\title{
Finite-size fluctuations and photon statistics near the polariton condensation transition in a single-mode microcavity
}

\author{
P. R. Eastham and P. B. Littlewood \\ Theory of Condensed Matter Group, Cavendish Laboratory, Madingley Road, Cambridge CB3 OHE, United Kingdom
}

(Received 31 October 2005; published 6 February 2006)

\begin{abstract}
We consider polariton condensation in a generalized Dicke model, describing a single-mode cavity containing quantum dots, and extend our previous mean-field theory to allow for finite-size fluctuations. Within the fluctuation-dominated regime the correlation functions differ from their (trivial) mean-field values. We argue that the low-energy physics of the model, which determines the photon statistics in this fluctuation-dominated crossover regime, is that of the (quantum) anharmonic oscillator. The photon statistics at the crossover are different in the high-temperature and low-temperature limits. When the temperature is high enough for quantum effects to be neglected we recover behavior similar to that of a conventional laser. At low enough temperatures, however, we find qualitatively different behavior due to quantum effects.
\end{abstract}

DOI: 10.1103/PhysRevB.73.085306

PACS number(s): 71.36.+c, 03.75.Hh, 42.50.Pq

\section{INTRODUCTION}

Microcavity polaritons ${ }^{1-5}$ are quasiparticles which form in wavelength-scale optical cavities containing dielectrics. They are mixed modes formed from cavity photons and dielectric excitations such as excitons. Since they are part photon polaritons are bosons, and are thus candidates for Bose condensation.

Experimental results on pumped microcavities continue to be linked to polariton condensation. ${ }^{6-9}$ The basic result is a threshold behavior of the luminescence intensity from a driven microcavity, while other features seen include nonthermal correlation functions for this luminescence, along with spatially and spectrally localized emission.

The central characteristic of Bose condensation is the generation of many-particle coherences which, in polariton condensation, appear in the electromagnetic field. The existence of polariton condensation remains controversial because polariton condensation is not the only phenomenon we can associate with coherent photons in microcavities. Most straightforwardly, if the cavity is driven into the weakcoupling regime one expects conventional lasing, and this is thought to be the correct interpretation of early claims for polariton condensation. ${ }^{10,11}$ While the more recent experiments cannot be straightforwardly attributed to conventional lasing, more exotic alternatives to polariton condensation, such as polaritonic lasing, ${ }^{12}$ remain.

While there may appear to be many different routes to optical coherence in microcavities, the relationships among these routes are not clear. Polariton condensation, polariton lasing, and conventional lasing are often assumed to be fundamentally distinct, but could equally well be related phenomena in different parameter regimes. This view is supported by recent work showing that adding decoherence processes to a mean-field theory of polariton condensation ${ }^{13-15}$ leads to a crossover from condensation to conventional lasing. ${ }^{16,17}$ It is also suggested by the fundamental connections between equilibrium and nonequilibrium phase transitions. The best known of these connections is between laser theory and the Landau theory of second-order phase transitions, ${ }^{18,19}$ but we note also recent work connecting the critical behavior of parametric oscillators and ferromagnets ${ }^{20}$ and a treatment of the ideal Bose gas along the lines of laser theory. $^{21}$

In this paper we investigate fluctuations close to polariton condensation, and how they affect the photon statistics. In general, there are different regimes for the dominance of fluctuations: in a very large system at low density of excitation the thermal equilibrium transition is of the BoseEinstein variety, ${ }^{22,23}$ so that spatial fluctuations are important. But since the polariton mass is very small (because of the large ratio between the wavelength of light and the typical exciton radius and exciton separation), at modest densities a BCS-like mean-field regime occurs and spatial fluctuations are small. Here the dominant fluctuations may be due to the finite size - or generically the finite population-of the system. This is very often also the case for conventional lasers ${ }^{18}$ (for similar reasons).

Here we consider finite-size fluctuations in isolation, by studying a single-mode model microcavity. We obtain an approximate form for the free energy of the model by neglecting quantum effects. This form can be interpreted as the classical probability distribution for the intensity of the cavity field and used to obtain all the static correlation functions of the cavity photons. It is identical to the accepted form for the intensity distribution near the onset of lasing, so we argue that within our approximations polariton condensation and lasing are not distinguished by the qualitative behavior of the static correlation functions. The parameters in the intensity distribution are associated with different physics in the two theories, so there remains room for quantitative distinctions between them.

While neglecting quantum effects in a theory of condensation leads to the same intensity distribution predicted by classical laser theory, this approximation fails for a condensate at low temperatures. When the temperature becomes comparable with or less than the interaction energy of two photons the finite level spacing affects the correlation functions. We shall see that this leads to behavior for the correlation functions of a condensate at low temperature which is 
qualitatively different from the predictions of standard laser theory. This difference is not surprising because standard laser theory is a classical approximation, controlled by the photon number at threshold.

Our analysis concerns the thermal equilibrium of a simplified model of a microcavity. Although in some parameter regimes current experiments are far from thermal equilibrium, there are several reasons to study the equilibrium behavior. The above-threshold luminescence in some recent experiments ${ }^{6}$ is suggestive of thermal equilibrium, and as microcavities continue to develop ${ }^{24,25}$ experiments can be expected to reach states closer to thermal equilibrium. Furthermore, the behavior close to equilibrium is expected to be similar to that in equilibrium, and an understanding of the equilibrium physics provides the basis for developing nonequilibrium theories. Finally, the qualitative behavior we exhibit here, in particular the functional forms of the correlation functions, derives from the structure of the effective theory describing the collective variables. This structure may be independent of whether the theory describes an equilibrium system such as a conventional condensate or a nonequilibrium system such as a conventional laser.

The remainder of this paper is organized as follows. We begin with an outline of the main results in Sec. II, which compares the classical laser, the classical and quantum critical fluctuations of the polariton condensate in terms of the anharmonic oscillator. The rest of the paper provides a derivation of these results, and a deeper quantitative analysis of the model.

In Sec. III we present the model we consider for both lasing and condensation, and give some necessary background on the mean-field theory of polariton condensation. Section IV contains the general analysis resulting in the free energy in the transition region, including a discussion of the regime of applicability of the classical approximation. In Sec. V we use these general results to analyze the phase diagram and photon statistics of the condensate. In Sec. VI we briefly review the standard calculations of the intensity distribution in a laser near threshold and compare with our results for the polariton condensate. In doing so, we note that conventional laser theory and its approximation to a phase transition relies on a "large- $N$ " justification that is not usually exposed. Section VII contains numerical estimates of the size of the fluctuation-dominated and quantum regimes in current condensation experiments. In Sec. VIII we discuss prospects for systems with large quantum regimes, and the role of spatial fluctuations. Finally, Sec. IX summarizes our conclusions.

\section{OUTLINE OF MAIN RESULTS: THE ANHARMONIC OSCILLATOR}

We shall find that the classical laser and quantum/classical condensate lie in the same universality class as the anharmonic oscillator

$$
H=\alpha \phi^{\dagger} \phi+\gamma \phi^{\dagger} \phi^{\dagger} \phi \phi .
$$

We choose $\phi$ to be normalized to obey the canonical Bose commutation relation $\left[\phi, \phi^{\dagger}\right]=1$.
The eigenstates of (1) are just the number states, $|n\rangle$, with energies $E(n)=(\alpha-\gamma) n+\gamma n^{2} \approx \alpha n+\gamma n^{2}$. Thus the partition function is

$$
Z=\sum_{n=0}^{\infty} e^{-\beta\left(\alpha n+\gamma n^{2}\right)},
$$

with $\beta=1 / k_{B} T$.

The parameter $\alpha$ is the tuning parameter through the transition. At the mean-field level, minimization of the exponent in (2) leads to

$$
\begin{gathered}
n_{\min }=0 \quad \text { for } \alpha>0, \\
n_{\min }=\frac{|\alpha|}{2 \gamma} \quad \text { for } \alpha<0 .
\end{gathered}
$$

Expanding in terms of fluctuations, $\delta n=n-n_{\min }$, one obtains (on the "condensed" side $\alpha<0$ ) quadratic number fluctuations controlled by $\gamma(\delta n)^{2}$. We shall choose the parameter $\gamma \propto 1 / N$, with $N$ growing with the system size; the limit $N \rightarrow \infty$ gives the mean-field result. We show below that the Dicke model indeed gives rise to a partition function of the form of (2) after truncating higher order terms in the exponent that are systematically smaller in powers of $1 / N$ (where in this case $N$ is the number of quantum dots in the cavity).

The summation in the partition function is of course still over discrete quantum states $|n\rangle$, but if the temperature is high enough the discreteness is irrelevant and the sum can be replaced by an integral. We now use fields $\psi=\phi / \sqrt{N}$ rescaled by system size so that $\psi$ appropriately describes the classical electromagnetic field intensity. Then one may write the partition function as

$$
Z \approx \int d \psi d \psi^{*} e^{-\beta N\left[\alpha|\psi|^{2}+\gamma N|\psi|^{4}\right]}
$$

[remembering that $\gamma N=O(1)]$. We derive this form explicitly for the Dicke model in Sec. IV. We now remark that the action in (4) is consistent with the steady-state distribution from a Fokker-Planck equation for diffusion in a quartic potential (see Sec. VI), which is the conventional approach to laser theory. Of course the "temperature" there is a fiction that is generated by couplings to (Markov) baths representing the outside world. Furthermore, there is no quantum limit for that type of laser theory. In the classical limit, the steady state laser and the polariton condensate have the same scaling form.

The anharmonic oscillator thus encapsulates the basic results of the two different models when we focus on the low energy physics. One signature of the transition is the intensity-intensity correlation function $g^{(2)}(0)$, which is straightforward to calculate for the anhmarmonic oscillator

$$
g^{(2)}(0)=\frac{\sum_{n} n(n-1) e^{-\beta[\alpha n+\gamma n(n-1)]}}{\left(\sum_{n} n e^{-\beta[\alpha n+\gamma n(n-1)]}\right)^{2}} .
$$

In Fig. 1 we plot $g^{(2)}(0)$ calculated numerically for (1), as a function of $\alpha$, at several temperatures. The tuning parameter $\alpha$ is proportional to density (at fixed temperature) for the 


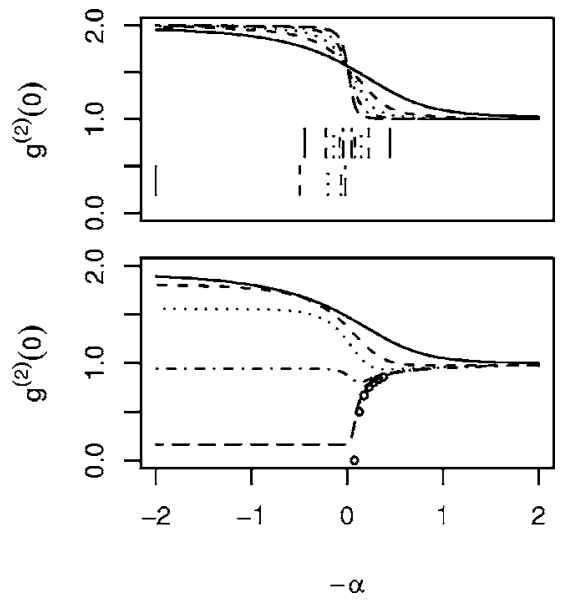

FIG. 1. $g^{(2)}(0)$ as a function of $\alpha$ for the thermal equilibrium of the anharmonic oscillator (1). The top panel shows the predictions of the classical approximation, while the bottom panel is the result of numerically evaluating the partition function. $\gamma=1 / 40$, and $T=2,1 / 2,1 / 5,1 / 15$, and $1 / 50$ for the solid, dashed, dotted, dotdashed, and long-dashed curves, respectively. The upper family of lines in the top panel are at $4(\gamma \beta)=(\alpha \beta)^{2}$, indicating the boundary of the classical fluctuation-dominated regime. The lower family are $\alpha=T$, indicating the range of validity of the classical approximation in the normal regime. The circles on the lower panel are the (discrete) values taken by $g^{(2)}(0)$ at $T=0$.

polariton problem, and generically is the pump rate in the laser model. The mean-field ordered phase is to the right, where we recover asymptotically $g^{(2)}(0) \rightarrow 1$ as expected for a classical macroscopic field. In the top panel, the classical (high-temperature) result is shown, with different curves corresponding to different temperatures. As temperature is lowered the result approaches more and more closely the meanfield theory, which is of course a step function. For specificity we have chosen $\gamma=1 / N=1 / 40$ for this demonstration, though the qualitative evolution will be the same for different values of $\gamma$.

While at high temperatures $(\gg \gamma) g^{(2)}(0)$ resembles the classical form, it is very different at low temperatures, $\lesssim \gamma$. Well below the transition the dominant contribution to $g^{(2)}(0)$ describes the thermal excitations of one and two photon states, so

$$
g^{(2)}(0) \approx 2 e^{-2 \beta \gamma}
$$

Although in this regime the statistics are strongly subPoissonian, the intensity is also very low, $\approx e^{-\beta \alpha}$. On crossing the transition the intensity increases and $g^{(2)}(0)$ approaches 1. At the lowest temperature $g^{(2)}(0)$ is a small constant below the transition, which then approximately follows the zero-temperature result above the transition: $g^{(2)}(0)=1-1 / n$ with $n$ the nearest integer to $-\alpha /(2 \gamma)-1 / 2$. At higher temperatures the quantum corrections decrease, and the form of $g^{(2)}(0)$ is intermediate between the classical result and the low-temperature one.

While the results are straightforwardly exposed in terms of the anharmonic oscillator, the mapping of the polariton condensate to the parameters $\alpha$ and $\gamma$ is more complex. The

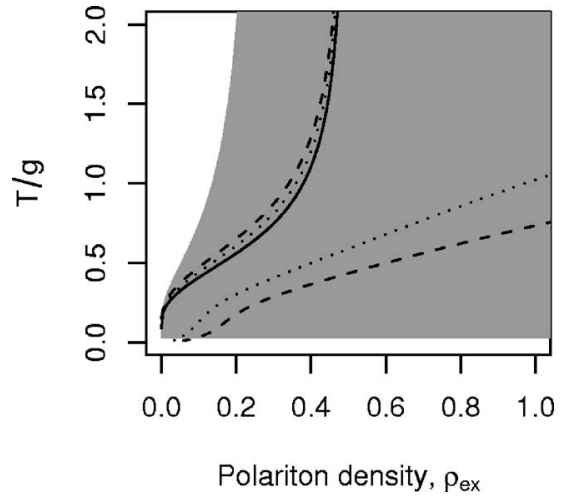

FIG. 2. Fluctuation-dominated regimes in terms of density and temperature for the polariton condensation transition in the model (7), with $E(i)=\omega$ so that all excitons are resonant with the cavity mode. The solid line is the mean-field boundary, and dashed (dotted) lines mark the boundaries of the fluctuation-dominated regions for $N=10$ (40). Curves correspond to those in Fig. 3. The shading indicates the regions in which the static approximation holds for $N=10$.

essential details are exposed in the phase diagram of Fig. 2.

The solid line is the mean-field phase boundary between the uncondensed (to the left) and condensed phases. Here the polariton density is measured per site, which explains why the mean-field boundary asymptotes to $\rho_{\mathrm{ex}}=1 / 2$ (the boundary of inversion) at large temperatures. The dashed and dotted lines mark regimes on either side of the mean-field transition where we estimate fluctuations to be substantial. These lines correspond to the upper panel of lines in Fig. 1. The strong asymmetry of the fluctuation regime about the meanfield line arises because we have chosen to expose the results with density as a parameter, rather than an external tuning parameter (here, naturally, the chemical potential). On the condensed side of the transition the number fluctuations are large because the chemical potential for polaritons becomes nearly clamped. The shading marks the estimated regime of validity of the classical approximation (akin to the lower panel of lines in Fig. 1). Over most of the figure the shaded regime encloses the fluctuation regime, and the classical approximation [which guarantees that $g^{(2)}(0)>1$ ] holds. For small densities and temperatures, however, we see that there is a strongly interacting quantum regime.

\section{MODEL}

Mean-field theories of polariton condensation have now been developed from models of a range of systems, including those with propagating photons. ${ }^{22,23}$ Here we consider the simplest model which leads to a theory of polariton condensation, the generalized Dicke ${ }^{26}$ model

$$
H=\omega \psi^{\dagger} \psi+\sum_{i} \frac{E(i)}{2} S_{i}^{z}+\frac{g}{\sqrt{N}} \sum_{i}\left(S_{i}^{+} \psi+\psi^{\dagger} S_{i}^{-}\right) .
$$

This is the basis of standard laser theory, as well as of our earliest mean-field theory ${ }^{13-15}$ of polariton condensation. It directly describes a system of localized excitons, for example 
in quantum dots, embedded in a three-dimensional microcavity. There is a single cavity mode, with annihilation operator $\psi$ and energy $\omega$, dipole coupled to a set of $N$ quantum dots or atoms of the gain medium. The state $S_{i}^{z}=+(-) 1$ corresponds to the presence (absence) of an exciton on site $i$, or to an atom in the upper (lower) of the states coupled by the lasing transition.

We have included a factor of $1 / \sqrt{N}$ explicitly in the lightmatter coupling in (7), so that for a cavity mode coupled to $N$ dots $g$ is related to the observed Rabi splitting and not to the single-dot Rabi splitting. This scaling is formally necessary because we will be concerned with the properties of the model (7) for large $N$, so we need the model to be well behaved in the limit $N \rightarrow \infty$. But we stress that, with our convention, changing the number of dots in a cavity of fixed volume corresponds to changing both $N$ and $g$.

To construct a theory of Bose condensation of polaritons from (7) one studies its thermodynamics, fixing the total number of excitons and photons

$$
N_{\mathrm{ex}}=\psi^{\dagger} \psi+\frac{1}{2} \sum_{i}\left(S_{i}^{z}+1\right) .
$$

Thus we consider the free energy, which can be written as the functional integral

$$
\frac{F}{k T}=f=\ln \int \mathcal{D} \psi \mathcal{D} \bar{\psi} e^{-N S_{\mathrm{eff}}},
$$

where

$$
S_{\mathrm{eff}}=\int_{0}^{\beta} d \tau \bar{\psi}\left(\partial_{\tau}+\widetilde{\omega}_{c}\right) \psi-\ln \operatorname{det} P .
$$

Here $\psi$ is related to the real electromagnetic field amplitude $\psi_{0}$ by $\psi_{0}=\psi \sqrt{N}$, while $\ln \operatorname{det} P$ corresponds to the free energy of the quantum dots in the field $\psi(\tau)$.

Equation (9) is the free energy in the grand-canonical ensemble. The constraint on $N_{\text {ex }}$ is dealt with on average, by introducing a chemical potential $\mu$ which appears in (10) as a shift of the photon and exciton energies: $\omega \rightarrow \widetilde{\omega}=(\omega-\mu)$ and $E(i) \rightarrow \widetilde{E}(i)=(E(i)-\mu)=2 \widetilde{\varepsilon}_{i}$. In the limit $N \rightarrow \infty$ the relative fluctuations of $N_{\mathrm{ex}}$ which occur in the grand-canonical ensemble are negligible, and the grand-canonical and canonical ensembles are expected to be equivalent. However, we are now considering fluctuations in a finite system, for which the predictions of the canonical and grand-canonical ensembles could differ. The correct ensemble then depends on how the polariton number is constrained experimentally. We consider an idealized limiting case in which this is done by coupling to a reservoir, so that the grand-canonical ensemble is appropriate.

We can develop an asymptotic expansion of the free energy for large $N$ by expanding $S_{\text {eff }}$ around its static saddle points $\psi(\tau)=\psi$. The saddle-point equation gives the meanfield estimate of the phase boundary between the normal state $(\psi=0)$ and the condensed state ( $\psi$ finite). Evaluating (9) on the stable saddle point leads to the mean-field estimate of the free energy; this is the only extensive contribution to the free energy, so that the mean-field theory gives the exact asymptotic form for the free energy in the thermodynamic limit $N \rightarrow \infty$.

Polariton condensation in the model (7) can be viewed as a generalization of the ferroelectric transition discovered by Hepp and Lieb. ${ }^{14,27}$ There has been recent interest in the physics associated with that ferroelectric transition in a finite model at zero temperature. ${ }^{28,29}$ This differs from the present problem in that there is no constraint on the polariton number, i.e., $\mu=0$, and as a consequence the rotating-wave approximation implicit in (7) cannot be made. This leads to qualitative differences ${ }^{28}$ in the dynamics of the unconstrained models at $T=0$.

\section{GENERAL FORM OF THE FREE ENERGY NEAR THE TRANSITION}

Away from the mean-field phase transition the saddlepoint expansion provides a systematic approximation scheme for the free energy of the finite system. However, this scheme fails in the vicinity of the mean-field transition due to the diverging occupations of the soft fluctuations. In this section we shall calculate the general form of the free energy of a large but finite system near the mean-field transition. To simplify the notation we shall take all the excitons to have the same energy, $E(i)=E_{g}$, but the resulting forms may be straightforwardly generalized to allow for a distribution of exciton energies.

We consider the action obtained by expanding (10) to fourth order in $\psi$,

$$
\begin{aligned}
S_{\text {eff }}= & S_{0}+\beta \sum_{\omega} \bar{\psi}(\omega) \psi(\omega) \frac{\left(i \omega+E_{+}\right)\left(i \omega+E_{-}\right)}{(i \omega+2 \widetilde{\varepsilon})} \\
& +\frac{g^{4}}{2} \sum_{\omega_{1}+\omega_{2}=\omega_{3}+\omega_{4}} \bar{\psi}_{\omega_{1}} \bar{\psi}_{\omega_{2}} \psi_{\omega_{3}} \psi_{\omega_{4}} V_{\omega_{1} \omega_{2} \omega_{3} \omega_{4}}+\cdots
\end{aligned}
$$

$S_{0}$ is the action of the two-level systems in the absence of photons, while the remaining part describes photons in the medium of the two-level systems. There are resonances at $E_{ \pm}$, which are the polariton energies relative to the chemical potential

$$
E_{ \pm}=\frac{1}{2}\left[\widetilde{\omega}_{c}+2 \widetilde{\varepsilon} \pm \sqrt{\left(\widetilde{\omega}_{c}-2 \widetilde{\varepsilon}\right)^{2}+4 g^{2} \tanh (\beta \widetilde{\varepsilon})}\right],
$$

while $V_{1234}$ is the photon-photon interaction mediated by the two-level systems

$$
\begin{aligned}
V_{1234}= & \sum_{\omega_{f}}\left\{\left(i \omega_{f}-\widetilde{\varepsilon}\right)^{-1}\left[i\left(\omega_{f}+\omega_{3}\right)+\widetilde{\varepsilon}\right]^{-1}\right. \\
& \times\left[i\left(\omega_{f}+\omega_{3}-\omega_{2}\right)-\widetilde{\varepsilon}\right]^{-1} \\
& \left.\times\left[i\left(\omega_{f}+\omega_{4}+\omega_{3}-\omega_{2}\right)+\widetilde{\varepsilon}\right]^{-1}\right\} .
\end{aligned}
$$

$\omega_{1}$, etc. are bosonic Matsubara frequencies, while $\omega_{f}=(n+3 / 4) 2 \pi T$ is a fermionic frequency, shifted to take account of the two-level constraint. ${ }^{23,30}$

\section{A. Static free energy}

As we approach the transition from the normal side one of the polariton energies $E_{ \pm}$is approaching zero, and perturba- 
tion theory fails. Over most of this fluctuation regime, however, $\omega_{1}=2 \pi T$ is large compared with the energy of the soft mode. The dominant contribution to the free energy for a large system close to the transition then comes from the static (classical) paths. Retaining only these dominant contributions gives an approximation for (9) near the transition,

$$
\begin{aligned}
f & \approx \ln \int d \psi d \psi^{*} e^{-N\left(\beta \tilde{\omega}_{c}|\psi|^{2}-\ln \cosh \beta \sqrt{\tilde{\varepsilon}^{2}+g^{2}|\psi|^{2}}\right)} \\
& =f_{0}+\ln \int d \psi d \psi^{*} e^{-N\left(a|\psi|^{2}+b|\psi|^{4}+c|\psi|^{6}+\cdots\right)} .
\end{aligned}
$$

Here $a=\beta E_{+} E_{-} /(2 \widetilde{\varepsilon})$ is the static part of the Gaussian kernel in (11), measuring the distance to the transition, $b$ is the static part of the interaction term, and $c, d, \ldots$ are higherorder interaction strengths which do not depend on the system size $N . f_{0}=N \ln \cosh \beta \widetilde{\varepsilon}$ is the free energy of the twolevel systems in zero field. The explicit form for $b$, obtained either from (13) or by expanding the exponent in (14), is

$$
b=g^{4} \beta \frac{\tanh (\beta \widetilde{\varepsilon})-\beta \widetilde{\varepsilon} \operatorname{sech}^{2}(\beta \widetilde{\varepsilon})}{8 \widetilde{\varepsilon}^{3}} .
$$

\section{B. Reduction to an oscillator}

At low temperatures the nonperturbative regime may lie outside the regime of validity of the static approximation, so that time-dependent paths must be considered. The divergences of perturbation theory are still at small frequencies, however, so for a large system close to the transition we can replace the action with its low frequency form. Considering for definiteness the region near the transition where $E_{-}$vanishes, we have $\omega, E_{-}$as small parameters, while generically $E_{+}$and $\widetilde{\varepsilon}$ are finite. The Gaussian term in (11) can then be straightforwardly approximated as

$$
\frac{\beta E_{+}}{2 \widetilde{\varepsilon}} \sum_{\omega} \bar{\psi}(\omega) \psi(\omega)\left(i \omega+E_{-}\right),
$$

with corrections proportional to the ratios of small to finite parameters. Approximating the interaction term is more involved, because the result of the summation (13) takes different forms depending on how many of the external frequencies coincide. This problem can be avoided by restricting our attention to the low-temperature regime $T \ll \widetilde{\varepsilon}$, in which all the forms lead to the same low-frequency approximation

$$
\frac{g^{4} \beta}{8 \widetilde{\varepsilon}^{3}} \sum_{\omega_{1}+\omega_{2}=\omega_{3}+\omega_{4}} \bar{\psi}_{1} \bar{\psi}_{2} \psi_{3} \psi_{4} .
$$

The corrections to (18) are again small in terms of the ratios of small to large parameters, e.g., $\omega / \widetilde{\varepsilon}$ and $T / \widetilde{\varepsilon}$. Since the coefficient in (18) is the low-temperature limit of $b$, it is convenient to replace it with $b$, leading to a low-energy approximation to the original theory which is valid at the quantum level for low temperatures, and at the classical level at higher temperatures.
Equations (17) and (18) become, after rescaling the fields $\psi \rightarrow \psi \sqrt{2 \widetilde{\varepsilon} / E_{+} N}$, the action for a quantum anharmonic oscillator (1). The oscillator frequency $\alpha=E_{-}$, and the interaction strength is $(b / \beta N)\left(2 \widetilde{\varepsilon} / E_{+}\right)^{2}$, or $g^{4} / 2 \widetilde{\varepsilon} N E_{+}^{2}$ at low temperatures.

\section{Validity of the static approximation}

The static approximation leading to (14) holds because the energy of fluctuations is much less than temperature. On the normal side of the transition this only occurs in a region close to the transition. However, the behavior of the excitation spectrum on the condensed side is different. This spectrum ${ }^{13-15}$ comprises a mode which is always at zero frequency relative to $\mu$, and two modes which at the transition are at positive and negative of the nonvanishing polariton energy. Thus there appears to be no mode which becomes soft at the transition, and so the classical approximation does not appear to be controlled by the distance from the transition.

This puzzle is resolved by inspecting the anharmonic oscillator (2), which we introduced earlier in Sec. II. On the normal side of the transition the nonlinear terms in (2) are irrelevant, and the parameter controlling the classical approximation is $\beta \alpha$. On the condensed side the exponent in (2) can be rewritten in terms of the number fluctuations $\delta n=n-n_{\min }=n-[-\alpha /(2 \gamma)]$ as $-\beta \gamma(\delta n)^{2}$. Thus the parameter controlling the static approximation on the condensed side is $\beta \gamma$. Returning to the polariton model close to the transition where $E_{-}$vanishes, we see that the classical approximation holds for the low-energy fluctuations when

$$
\frac{b}{N}\left(\frac{2 \widetilde{\varepsilon}}{E_{+}}\right)^{2} \ll 1
$$

provided the temperature is small compared with the Rabi splitting and $\widetilde{\varepsilon}$. At higher temperatures we expect an inequality of the same general form, $T N / g \gtrsim 1$, but with numerical differences due to the renormalization of the effective interaction by the occupation of the high-energy polariton and the frequency dependence of the interaction.

Our previous computations ${ }^{13-15}$ of the fluctuation spectrum were done at a Gaussian level, i.e., approximating the partition function of the fluctuations with that of a harmonic oscillator. This approximation predicts that the characteristic frequency of fluctuations about the condensate is zero because there is no linear term in $\delta n$ when the exponent of (2) is expanded around a finite $n$. To obtain a finite level spacing one must go beyond the Gaussian approximation and include interactions, which generate a finite level spacing $\sim 1 / N$. This may be contrasted with the normal state, where the harmonic oscillator part of (2) is enough to generate a finite level spacing $\alpha$.

\section{CORRELATION FUNCTIONS AND PHASE DIAGRAM}

The correlation functions of the cavity field can be obtained by differentiating (14) with respect to $\widetilde{\omega}_{c}$ or (15) with respect to $a$. Note that the field is classical: the integrand of (14) can be interpreted as a probability distribution for the 
intensity of the cavity field. Nonclassical fields are associated with time-dependent paths, which give complex integrands in the path integral (9) that cannot be interpreted as classical probabilities.

The integral (14) is only tractable numerically. However, the interactions $c, d, \ldots$ do not affect the asymptotic behavior of the correlation functions as $N \rightarrow \infty$ if $a \geqslant 0$ and $b>0$, because the quartic nonlinearity restricts fluctuations of the field to $|\psi| \lesssim N^{-1 / 4} b^{-1 / 4}$. Therefore, we can obtain the asymptotic forms of the correlation functions on the normal side of the mean-field phase boundary from (15) with $c, d, \ldots=0$. The corresponding free energy is, discarding additive constants,

$$
\begin{aligned}
f & =\ln \int d \psi d \psi^{*} e^{-N\left(a|\psi|^{2}+b|\psi|^{4}\right)} \\
& =\ln \frac{e^{N a^{2} / 4 b} \operatorname{erfc}\left(\frac{a}{2} \sqrt{\frac{N}{b}}\right)}{\sqrt{b N}} .
\end{aligned}
$$

On the condensed side where $a<0$ we may still use the approximation (20) so long as the order parameter $|\psi|$ is small. It is a weaker approximation than on the normal side, because the minimum of the exponent in (15) occurs for $|\psi|^{2} \sim 1$, so that truncation to a quartic theory produces errors in the leading asymptotics of the correlation functions. However, these errors are proportional to the order parameter, and so are numerically small close enough to the transition, even if they are not asymptotically small in $N$.

We now present explicit results for the behavior of the cavity field in the model (9) when $N$ is finite but large. For orientation, the mean-field phase boundaries $E_{ \pm}=0$ are shown as the solid lines in Fig. 2. $\rho_{\mathrm{ex}}$ is the number of polaritons per site, $\left\langle N_{\mathrm{ex}} / N\right\rangle$ with $N_{\mathrm{ex}}$ given by (8); note that this differs from the definition of $\rho_{\text {ex }}$ used in Refs. $13-15$ by a shift of 0.5. Temperature is expressed in units of $g$, which is one-half of the collective Rabi splitting at resonance [see Eq. (12)].

For completeness we begin by considering the region far from the transition, where the correlation functions can be obtained using the saddle-point expansion. On the normal side of the mean-field transition there is no saddle-point contribution to the photon density $\left\langle\psi^{\dagger} \psi\right\rangle$, so the first nonvanishing contribution is at order $1 / N$. This term is just the expectation value of the photon density from the Gaussian part of (11), i.e., the photon density in a population of noninteracting polaritons with energies $E_{ \pm}$:

$$
\begin{gathered}
\left\langle\psi^{\dagger} \psi\right\rangle=\frac{\left(E_{+}-2 \widetilde{\varepsilon}\right) n_{\mathrm{B}}\left(E_{+}\right)+\left(2 \widetilde{\varepsilon}-E_{-}\right) n_{\mathrm{B}}\left(E_{-}\right)}{N\left(E_{+}-E_{-}\right)}, \\
n_{\mathrm{B}}(x)=\frac{1}{e^{\beta x}-1} .
\end{gathered}
$$

Since at this order in $N$ we have noninteracting particles, the many-photon correlation functions are related to the photon number by Wick's theorem. In particular, for the static part

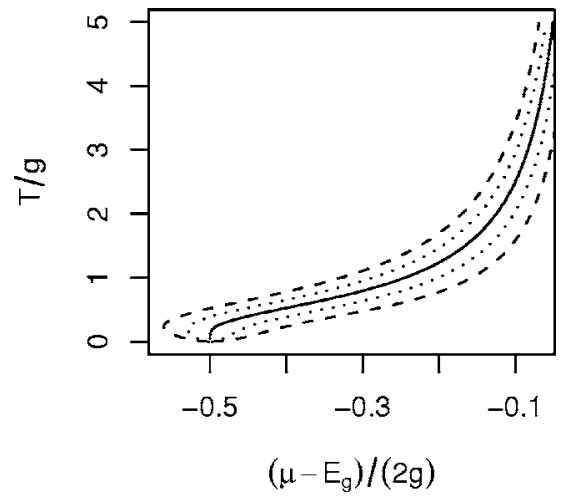

FIG. 3. Fluctuation-dominated regimes for the polariton condensation transition with $\Delta=0$. Solid lines are the mean-field boundaries. Dashed (dotted) lines mark the boundaries of the fluctuationdominated regions for $N=10(40)$.

of the correlation function measured by Deng et al. we have $g^{(2)}(0)=\left\langle\psi^{\dagger} \psi^{\dagger} \psi \psi\right\rangle /\left\langle\psi^{\dagger} \psi\right\rangle^{2}=2+O(1 / N)$.

On the condensed side of the mean-field transition the leading-order contributions to the correlation functions come from the saddle point. Thus $\left\langle\psi^{\dagger} \psi\right\rangle \sim 1$, and we expect $g^{(2)}(0)=1+O(1 / N)$. Calculations of the higher-order terms are complicated due to the presence of the zero mode, and so we shall not pursue them here.

In the region close to the mean-field transition the saddlepoint expansion must break down, so that the correlation functions can smoothly interpolate between their forms in the two states. We can estimate the boundaries of this crossover region by equating the magnitudes of successive terms in the large- $N$ expansion of the photon density, $\left\langle\psi^{\dagger} \psi\right\rangle$. For the approximate form (21) we see that the crossover regime obeys $N a^{2} \leqslant 4 b$, or

$$
\left|\frac{2 g^{4}}{\beta N \widetilde{\varepsilon} E_{+}^{2} E_{-}^{2}}\left[\tanh (\beta \widetilde{\varepsilon})-\beta \widetilde{\varepsilon} \operatorname{sech}^{2}(\beta \widetilde{\varepsilon})\right]\right| \geqslant 1 .
$$

Note that the numerical factor in this expression is not meaningful, since it depends on the precise definition of the boundaries of the crossover regime.

In Fig. 3 we show the phase diagram of the system as a function of $\mu$ and $T$, for $\Delta g=\omega-E_{g}=0$. The solid lines are the phase boundary of the infinite system, $E_{+} E_{-}=0$, while the remaining lines are the boundaries of the crossover regions (24) for systems of 10 and 40 quantum dots. One general feature which can be seen in this figure is that the size of the fluctuation regions scales as $1 / \sqrt{N}$, as can be deduced from (21). The particular shapes of the fluctuation regions come from the interplay between the thermal occupations and the strength of the interaction, which is temperature and density dependent. In particular, the present model has the unusual feature that the fluctuation regions vanish in the hightemperature limit, where the limits of the inequality (24) are approximately

$$
4 \widetilde{\varepsilon}=g^{2} \beta-2 \Delta g \pm \frac{2 \beta g^{2}}{\sqrt{3 N}} .
$$

Physically, this occurs because for large $T$ the number of fluctuations at fixed chemical potential behaves as 
$n_{\text {fluc }}=n_{\mathrm{B}}\left(E_{+}\right)+n_{\mathrm{B}}\left(E_{-}\right) \sim T$, while the interaction strength $b$ vanishes like $1 / T^{4}$. Thus the mean interaction energy $\left\langle n_{\text {fluc }}^{2} b\right\rangle$ vanishes in the high temperature limit. The occupation of the fluctuations diverges only as $T$ because they are confined to a finite energy range, so that increasing temperature does not increase the number of relevant fluctuation modes, as it would in the more familiar case of a semi-infinite band of states. The interaction vanishes because at high temperatures the field does not affect the occupation of the two-level systems: the free energy of a two-level system, which appears in the exponent of (14), becomes independent of field $|\psi|$ as $T \rightarrow \infty$.

Figure 2 shows the same phase diagram in terms of density and temperature, obtained by relating chemical potential to density using the mean-field results. For the normal state we use

$$
\rho_{\text {ex }}=\rho_{\text {excitons }}=[1-\tanh (\beta \widetilde{\varepsilon})] / 2,
$$

while for the condensate the quartic theory gives

$$
\rho_{\mathrm{ex}}=\rho_{\text {excitons }}+k T \frac{\partial}{\partial \mu}\left(\frac{a^{2}}{4 b}\right) \text {. }
$$

These relations introduce qualitative differences between the fluctuation-dominated regions in this figure and those in Fig. 3. In particular, the fluctuation-dominated region becomes much larger on the condensed side, because the chemical potential is only weakly dependent on density in the condensate.

The shading on Fig. 2 indicates the regimes of validity of the classical approximation, combining the analysis of Secs. IV B and IV C with Eqs. (26) and (27). On the normal side of the mean-field transition we shade the region $\beta E_{-}<1$. On the condensed side the analogous inequality is (19). However, the numerical factors in this result are only correct close to the phase boundary. Since this only occurs at low densities we have plotted the inequality $T / g>1 /(4 N)$, corresponding to the low-density limit of (19).

In the upper panel of Fig. 4 we plot the prediction of (21) for the number of photons in the cavity

$$
N\left\langle\psi^{\dagger} \psi\right\rangle=-\frac{\partial f}{\partial a},
$$

as a function of the deviation of $\mu$ from its mean-field critical value $\mu_{c}$. We take $\Delta=0$, and plot curves for $T / g=0.25,0.75$, and 1.5 , and for $N=10$ and 40 .

In general each of the $N$ two-level systems makes a contribution of order $1 / N$ to the number of cavity photons, due to the scaling of the coupling constant. Well below the transition we could neglect the interactions between the photons and approximate the integrand of (15) by a Gaussian. This gives $N\left\langle\psi^{\dagger} \psi\right\rangle \approx 1 / a$. In the Gaussian regime each two-level system contributes incoherently to the cavity field, so the total photon number is of order 1; such scaling is demonstrated by the collapse of the curves corresponding to different system sizes in the left side of the top panel of Fig. 4.

As we increase the chemical potential through the meanfield transition the occupancy of the cavity field increases, and the interactions begin to generate coherence between the
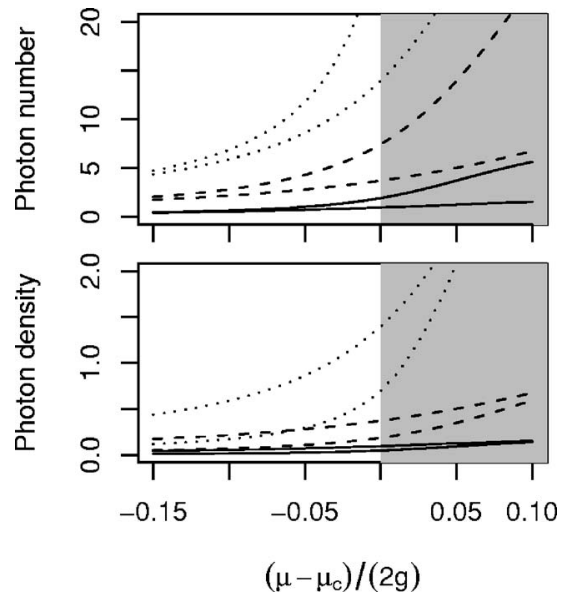

FIG. 4. Photon number $N\left\langle\psi^{\dagger} \psi\right\rangle$ (top panel) and density $\left\langle\psi^{\dagger} \psi\right\rangle$ (bottom panel) as functions of $\left(\mu-\mu_{c}\right) /(2 g)$ for $\Delta=0 . T / g=0.25$ (solid lines), 0.75 (dashed lines), and 1.5 (dotted lines). $N=10$ (lower line of each pair in the top panel, upper in the bottom panel) and $N=40$ (upper line in top panel, lower in bottom panel). Shading marks the condensed region of the mean-field theory.

contributions of the different two-level systems. Far in the condensed state this coherence is complete: all the $\sim N$ twolevel systems contribute coherently to the cavity field, giving a photon number which scales as $N$. This scaling can be seen on the right of the lower panel of Fig. 4, which shows the photon number per two-level system. The order of $N$ contribution to the photon number comes from the saddle point of (20), and is $-a /(2 b)$. This saddle-point contribution is the exponential in (21); it survives in the condensed state, but is canceled by the asymptotic expansion of the error function well into the normal state.

In the region near the transition neither the Gaussian nor saddle-point approximations are appropriate, and the full form (21) must be used. There is partial coherence amongst the two-level systems, leading to a photon number which scales as $\sqrt{N}$. Explicitly, we find $N\left\langle\psi^{\dagger} \psi\right\rangle=\sqrt{N /(\pi b)}$ for the photon number at the transition. The scaling can be shown from a more general argument by noting that the $a$ dependent part of (20) is a function of $\sqrt{N a}$ and $b$, so at the transition

$$
N\left\langle\psi^{\dagger} \psi\right\rangle=-\left.\frac{\partial f(\sqrt{N} a, b)}{\partial a}\right|_{a=0}=-\sqrt{N} g(0, b) .
$$

Figure 5 illustrates the results obtained from (21) for the static intensity-intensity correlation function

$$
g^{(2)}(\tau=0)=1+\frac{\frac{\partial^{2} f}{\partial a^{2}}}{\left(\frac{\partial f}{\partial a}\right)^{2}} .
$$

Well below the transition $g^{(2)}(0)$ approaches the value of 2 associated with a thermal mixture of noninteracting photons, while well above it $g^{(2)}(0)$ approaches 1 , corresponding to the bunched photons of, e.g., a coherent state or largeamplitude number state. The crossover occurs over a range of chemical potentials which scales as $1 / \sqrt{N}$. 


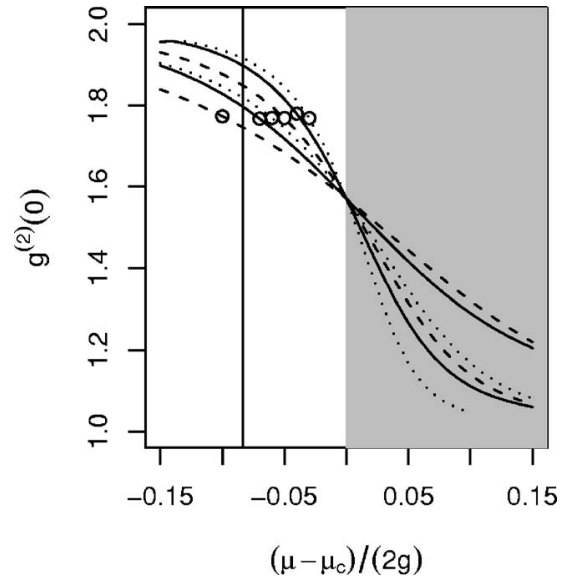

FIG. 5. Intensity-intensity correlation function, $g^{(2)}(0)$, of the cavity photons as a function of chemical potential, $\left(\mu-\mu_{c}\right) /(2 g)$, for $\Delta=0 . T / g=0.25$ (solid lines), 0.75 (dashed lines), and 1.5 (dotted lines). $N=10$ (lower line of each pair in the normal region) and $N=40$ (upper line). Shading marks the condensed region of the mean-field theory. Circles correspond to the lines marking the fluctuation-dominated regimes of the normal state in Fig. 2. The vertical line corresponds to the limit of the shading in the normal state on Fig. 2 for $T / g=0.25$; the analogous lines for $T / g=0.75$ and 1.5 are off the scale.

\section{LASER FLUCTUATIONS}

In this section, we briefly review the standard theory of fluctuations in a single-mode laser close to threshold, following the work of Risken. ${ }^{19}$ We shall see that the photon statistics predicted by this theory are similar to those predicted by the theory of polariton condensation. This similarity holds in spite of the fact that the theory of condensation describes a system in thermal equilibrium and laser theory one which is not in thermal equilibrium.

The canonical laser theories are based on the dynamics of models such as (7) in the presence of dissipation processes. Such dissipation processes can be formally generated by coupling each dot, and the photon mode, to an oscillator bath. Thus one considers Hamiltonians of the general form

$$
H_{T}=H+H_{\text {res }}+H_{\text {ph-res }}+H_{\text {s-res }},
$$

where $H=H_{\mathrm{ph}}+H_{\mathrm{s}}+H_{\mathrm{s}-\mathrm{ph}}$ is given by Eq. (7), $H_{\text {res }}$ describes the harmonic oscillator baths, and the remaining two terms couple the system and baths. The standard model is to couple the cavity mode to its bath with Hamiltonian

$$
H_{\mathrm{ph}-\mathrm{res}}=\sum_{p} g_{p}\left(\psi^{\dagger} d_{p}+d_{p}^{\dagger} \psi\right)
$$

and the dots to their baths with Hamiltonian

$$
H_{\mathrm{s}-\mathrm{res}}=\sum_{i} \sum_{p} g_{p i}^{\perp}\left(d_{p i}^{\dagger} S_{i}^{-}+S_{i}^{+} d_{p i}\right)+g_{i p}^{\|} d_{p i}^{\dagger} d_{p i} S_{z} .
$$

To develop dynamical equations for the system variables alone one takes the Liouville equation for the evolution of the density matrix and averages over the reservoir variables. The interaction between the system and reservoir variables is treated using second-order perturbation theory, leading to an integrodifferential equation for the time evolution of the reduced density matrix $\rho$. This equation is then approximated by a first-order differential equation, implicitly neglecting memory effects, formally by having Markovian baths whose spectra are completely white.

Finally, one integrates the atomic degrees of freedom out of the density matrix for the system, assuming that the atomic system is very strongly damped. This leads to an equation of motion for the $P$ representation of the field density matrix, $W\left(\psi, \psi^{*}, t\right)$. Neglecting terms associated with the quantum nature of the field, ${ }^{19}$ this equation becomes the Fokker-Planck equation describing diffusion in a quartic potential

$$
\frac{\partial W}{\partial t}+\zeta \frac{\partial}{\partial \psi}\left(d-|\psi|^{2}\right) \psi W+\zeta \frac{\partial}{\partial \psi^{*}}\left(d-|\psi|^{2}\right) \psi^{*} W=4 q \frac{\partial^{2} W}{\partial \psi \partial \psi^{*}} .
$$

The coefficients in this form are the strength of the nonlinearity $\zeta$ (denoted $\beta$ in Ref. 19), the linear gain or loss, $\zeta d$, and a diffusion constant $q$.

To compare with the theory of fluctuations near polariton condensation, we make the same rescaling of the field as we did there, $\psi \rightarrow \psi \sqrt{N}$. In terms of this rescaled field, the steady-state probability distribution obtained from (34) is proportional to the integrand of (20). The parameters are related to the laser parameters as $a=-\zeta d /(2 q)$, and $b=\zeta N /(4 q)$. The scaling of these terms follows from the microscopic expressions for $\zeta, q$, and $d$ given in Eqs. (2.17), (2.27) of Ref. 19. Substituting $g \rightarrow g / \sqrt{N}$ so that $g$ is as defined in Eq. (7), and noting that the total inversion $\sigma$ scales as $N$, we find $q \sim N^{0}, \zeta \sim N^{-1}, \zeta d \sim N^{0}$. Therefore, as in the polariton condensate, the parameters $a$ and $b$ are independent of $N$.

Thus in the classical approximation the photon statistics of a laser and a condensate differ only due to the dependence of $a$ and $b$ on the microscopic parameters of the system. This is similar to the usual universality which occurs close to a second-order phase transition, where the singular parts of the observables are given by universal scaling functions, with nonuniversal relations between the scaling parameters and physical parameters such as temperature. In the finite systems considered here the observables are not singular, but for large $N$ the correlation functions in the two problems are identical functions of a parameter describing the distance from the transition. This arises because the collective behavior of the electromagnetic field in both theories is described by a quartic "free-energy," and the system size $N$ enters these free energies in the same way.

Within our approximations, then, the only scope for making a distinction between polariton condensation and lasing arises from the forms of $a$ and $b$ in the two systems. These forms are model dependent and expected to change in more realistic models of either system. However, we note that there are differences in the physics of these forms in the laser and the condensate, which could be expected to be robust. For example, in the laser the noise strength $q$ is the spontaneous emission rate into the lasing mode, while in the condensate the analogous noise strength is temperature. It 
appears that the laser amplifies spontaneous emission noise, while the condensate amplifies thermal noise. The parameters $a$ and $b$ also contain different physics: in the laser, $a$ is associated with gain or loss, and $b$ with gain depletion, while in the condensate, $a$ is a single-particle energy, and $b$ an interaction strength. Thus the size of the threshold regime in absolute units is controlled by different physics in the two theories.

The analogy between the laser threshold and a secondorder phase transition is well known, and previous authors have pointed out that this analogy extends beyond the thermodynamic limit: ${ }^{18,31}$ in a finite system, the singularities associated with a phase transition are rounded in the same way that the sharp singularities of the lasing transition in a rateequation treatment are rounded by noise. We see that for the laser model (31) the parameter controlling this rounding is $N$, the system size, exactly as in the usual thermodynamic case. This appears to differ from the picture obtained from classical stochastic models, ${ }^{31}$ in which the corresponding parameter is the fraction of the spontaneous emission directed into the lasing mode.

\section{QUANTITATIVE ESTIMATES FOR CURRENT EXPERIMENTS}

We can use our model to obtain some indication of the scale of the fluctuation-dominated region in current experimental systems. Such systems are generally planar microcavities, rather than the three-dimensional cavity of (7). However, the luminescence which may be evidence for condensation is localized, suggesting that the condensate itself is localized, presumably due to some combination of kinetics, self-trapping, and disorder. In a condensate localized on scale $L$ we expect there to be an energy cost $\sim\left(L^{2} m_{p}^{2}\right)^{-1}$ associated with spatial variations, where $m_{p} \sim 10^{-5} m_{e}$ is the in-plane mass of the polaritons. If this energy cost is large compared with temperature we expect spatial fluctuations to be unimportant, and the present theory to apply.

Let us consider in particular the data discussed by Weihs, Deng, Snoke, and Yamamoto in Ref. 32. These experiments are done on a GaAs planar microcavity with twelve quantum wells and a Rabi splitting of $14.9 \mathrm{meV}$, so $g \approx 86 \mathrm{~K}$. Their condensate is typically $5 \mu \mathrm{m}$ in diameter, giving a temperature scale for spatial fluctuations of $60 \mathrm{~K}$. We take the Mott density, $10^{12} \mathrm{~cm}^{-2}$, to be an upper bound on the density of available exciton sites, so the condensate of diameter $5 \mu \mathrm{m}$ corresponds to $N \sim 10^{5}$.

The temperature in these systems is extremely low relative to the Rabi splitting: $T=5 \mathrm{~K}$ corresponds to $T / g=0.06$. At these low temperatures the critical density is not associated with the single-mode phase transition given here, but with spatial fluctuations, whose effect on the mean-field theory is analyzed in Refs. 22 and 23. Thus our theory cannot describe the transition that is being crossed in the experiments. However, once the condensate has formed we expect it to apply, since the condensate which forms is apparently localized, and the spatial degrees of freedom are frozen out. We estimate that the achieved condensate densities of $10^{10} \mathrm{~cm}^{-2}$ correspond to $\rho_{\mathrm{ex}} \approx 10^{10} / n_{\text {Mott }}=10^{-2}$. For this density the transition temperature of our theory is $T / g=0.2 \approx 20 \mathrm{~K}$. For $N=10^{5}$ the fluctuation-dominated region extends over around $20 \mathrm{mK}$ on the normal side of the transition and $1 \mathrm{~K}$ on the condensed side. The experimental temperature is far outside this region, so the present theory suggests that the fluctuations of the condensate are negligible and the light from the condensate area is almost perfectly coherent. If raising the temperature did not change the localization then this would allow the sharp crossover predicted here to be observed. This complication would be absent for systems with external in-plane confinement of the polaritons, such as pillar microcavities. ${ }^{33}$

For a system in thermal equilibrium, we have argued that the quantum regime occurs when the temperature is smaller than the characteristic level spacing produced by the photonphoton interaction. For the particular model used here, this is when $T N \sim g$. We estimate that this gives a scale of $1 \mathrm{mK}$ for the systems of Ref. 32, which is far below the achieved temperatures. Furthermore, these are open systems coupled to baths. In such a system, the effect of dissipation is loosely to broaden the level spectrum through the decay process. To have quantum effects that dominate for the longest times, one then requires linewidths that are narrow in comparison to the level spacing. This is not achieved, since a temperature scale of $1 \mathrm{mK}$ corresponds to a linewidth on the order of $10 \mu \mathrm{eV}$, far below the linewidth associated with the decay of the cavity mode.

\section{DISCUSSION}

Recently, three groups ${ }^{34-36}$ have achieved strong-coupling of single quantum dots in microcavities, and are thus beginning to approach the regime, already achieved in atom cavity optics, ${ }^{37}$ of a single two-level system strongly coupled to a cavity mode. Clearly the nonlinear regime ${ }^{38}$ of such a system is dominated by quantum effects. However, we note that our model suggests that this single-atom limit is not the only way to see strong quantum effects. For a cavity of fixed volume one should replace $g / \sqrt{N}$ with $g$ in (7). The effective photonphoton interaction then scales as $N$ (whereas it scales as $N^{-1}$ at fixed dot density), so that quantum effects survive to higher temperatures when the number of dots increases in a cavity of fixed volume: a situation opposite to the normal thermodynamic limit we consider elsewhere, in which quantum effects move to lower temperatures as the system size increases. In this context it is interesting to note the experiments of Ref. 39, in which the quantum statistical properties of light emitted from a weakly driven atom-cavity system are measured. The observed $g^{(2)}(0)$ was below the classical limit of 1 , and furthermore its value was apparently independent of the number of atoms in the cavity. This would naturally arise if the noise increased as $N$, as it does in standard laser theory, canceling the increase in the level spacing.

The behavior of a finite system in which spatial fluctuations are allowed could be analyzed using renormalization group arguments. ${ }^{40}$ We expect it to depend on the interplay between system size and an effective coordination number or interaction range: a small system with a large coordination (long-range interactions) will be dominated by finite-size 
fluctuations over a larger region of the phase diagram than it is dominated by spatial fluctuations, whereas the reverse will occur for a large system with a small coordination (shortrange interactions). In the former case we can view the finite-size fluctuations as corrections to mean-field theory, as here. This cannot be true in the latter case, however, since the theory of the finite system should then involve critical exponents different from those of mean-field theory.

The phase diagram of the infinite two-dimensional system, allowing for spatial fluctuations, has been studied in Ref. 23. The deviations between the phase boundary there and that of mean-field theory indicate the regions in which spatial fluctuations of an infinite system are significant. Since the photon mass is very small, effectively providing a longrange interaction between excitons, these deviations are only significant at very low densities, where even a long-range interaction gives a small coordination number. Hence, except at low densities, we expect even a relatively large system to have mean-field like finite-size behavior.

\section{CONCLUSIONS}

In this paper, we have analyzed the behavior of a model of a finite polariton condensate close to the mean-field transition and shown that it is formally similar to the theory describing a laser close to the laser threshold. This similarity fails when the polariton condensate reaches a lowtemperature quantum regime, since the laser theory is classical—notwithstanding that the noise in the laser theory might ultimately have its origins in quantum effects.

In the classical regime we find that the intensity distribution for the photons in a finite polariton condensate is of the same form as that obtained from conventional laser theory. Thus the photon statistics are not expected to reveal any fundamental difference between a condensate and a laser, but of course the parametrization is different and the scales have different meanings. In particular, for a condensate in thermal equilibrium the sharpness of the crossover is controlled by temperature, whereas in a laser it is controlled by the noise introduced by coupling to external baths.

Quantum effects dominate in general when the level spacing exceeds the noise strength. We have seen that for the polariton condensate in equilibrium the relevant level spacing is the photon-photon interaction, and the relevant noise strength is temperature. In the conventional thermodynamic limit of our model the temperature scale for quantum effects decreases with system size, but the opposite occurs when increasing the dot number with fixed cavity volume. Hence, it is not always the smallest systems which have the most strongly quantum-mechanical collective behavior.

\section{ACKNOWLEDGMENTS}

We thank Simon Kos, Vikram Tripathi, Jonathan Keeling, and Mike Gunn for helpful discussions of this work, and acknowledge support from the EPSRC, Sidney Sussex College, Cambridge, and the EU RTN Project No. HPRN-200200298.
${ }^{1}$ J. J. Hopfield, Phys. Rev. 112, 1555 (1958).

${ }^{2}$ C. Weisbuch, M. Nishioka, A. Ishikawa, and Y. Arakawa, Phys. Rev. Lett. 69, 3314 (1992).

${ }^{3}$ M. S. Skolnick, T. A. Fisher, and D. M. Whittaker, Semicond. Sci. Technol. 13, 645 (1998).

${ }^{4}$ V. Savona, in Confined Photon Systems, Lecture Notes in Physics, edited by H. Benisty, J. M. Gérard, R. Houdré, J. Rarity, and C. Weisbuch (Springer, Berlin, 1999), pp. 173-242.

${ }^{5}$ A. Kavokin and G. Malpuech, Cavity Polaritons, Thin Films and Nanostructures, Vol. 32 (Elsevier, London, 2003).

${ }^{6}$ H. Deng, G. wei, D. Snoke, J. Bloch, and Y. Yamamoto, Proc. Natl. Acad. Sci. U.S.A. 100, 15318 (2003).

${ }^{7}$ M. Richard, J. Kasprzak, R. Romestain, R. André, and L. S. Dang, Phys. Rev. Lett. 94, 187401 (2005).

${ }^{8}$ L. S. Dang, D. Heger, R. André, F. Boeuf, and R. Romestain, Phys. Rev. Lett. 81, 3920 (1998).

${ }^{9}$ P. Senellart and J. Bloch, Phys. Rev. Lett. 82, 1233 (1999).

${ }^{10}$ S. Pau, H. Cao, J. Jacobson, G. Björk, Y. Yamamoto, and A. Imamoğlu, Phys. Rev. A 54, R1789 (1996).

${ }^{11}$ X. Fan, H. Wang, H. Q. Hou, and B. E. Hammons, Phys. Rev. A 56, 3233 (1997).

${ }^{12}$ F. P. Laussy, G. Malpuech, A. V. Kavokin, and P. Bigenwald, J. Phys.: Condens. Matter 16, S3665 (2004).

${ }^{13}$ P. R. Eastham and P. B. Littlewood, Solid State Commun. 116, 357 (2000)

${ }^{14}$ P. R. Eastham and P. B. Littlewood, Phys. Rev. B 64, 235101
(2001).

${ }^{15}$ P. R. Eastham, Ph.D. thesis, University of Cambridge, 2000.

${ }^{16}$ M. H. Szymanska and P. B. Littlewood, Solid State Commun. 124, 103 (2002).

${ }^{17}$ M. H. Szymanska, P. B. Littlewood, and B. D. Simons, Phys. Rev. A 68, 013818 (2003).

${ }^{18}$ H. Haken, Rev. Mod. Phys. 47, 67 (1975).

${ }^{19}$ H. Risken, in Progress in Optics, edited by E. Wolf (NorthHolland, Amsterdam, 1970), Vol. 8, Chap. 5, pp. 241-294.

${ }^{20}$ P. D. Drummond and K. Dechoum, Phys. Rev. Lett. 95, 083601 (2005).

${ }^{21}$ M. O. Scully, Phys. Rev. Lett. 82, 3927 (1999).

${ }^{22}$ J. Keeling, P. R. Eastham, M. H. Szymanska, and P. B. Littlewood, Phys. Rev. Lett. 93, 226403 (2004).

${ }^{23}$ J. Keeling, P. R. Eastham, M. H. Szymanska, and P. B. Littlewood, Phys. Rev. B 72, 115320 (2005).

${ }^{24}$ T. Tawara, H. Gotoh, T. Akasaka, N. Kobayashi, and T. Saitoh, Phys. Rev. Lett. 92, 256402 (2004).

${ }^{25}$ A. Pawlis, A. Kharchenko, O. Husberg, K. Lischka, and D. Schikora, J. Phys.: Condens. Matter 16, S3689 (2004).

${ }^{26}$ R. H. Dicke, Phys. Rev. 93, 99 (1954).

${ }^{27}$ K. Hepp and E. H. Lieb, Ann. Phys. (N.Y.) 76, 360 (1973).

${ }^{28}$ C. Emary and T. Brandes, Phys. Rev. Lett. 90, 044101 (2003).

${ }^{29}$ J. Vidal and S. Dusuel, cond-mat/0510281 (unpublished).

${ }^{30}$ V. N. Popov and S. A. Fedetov, Zh. Eksp. Teor. Fiz. 94, 183 (1988) [Sov. Phys. JETP 67, 535 (1988)]. 
${ }^{31}$ P. R. Rice and H. J. Carmichael, Phys. Rev. A 50, 4318 (1994).

${ }^{32}$ G. Weihs, H. Deng, D. Snoke, and Y. Yamamoto, Phys. Status Solidi A 201, 625 (2004).

${ }^{33}$ K. J. Vahala, Nature (London) 424, 839 (2003).

${ }^{34}$ T. Yoshie, A. Scherer, J. Hendrickson, G. Khitrova, H. M. Gibbs, G. Rupper, C. Ell, O. B. Shchekin, and D. G. Deppe, Nature (London) 432, 200 (2004).

${ }^{35}$ J. P. Reithmaier, G. Sek, A. Löffler, C. Hofmann, S. Kuhn, S. Reitzenstein, L. V. Keldysh, V. D. Kulakovskii, T. L. Reinecke, and A. Forchel, Nature (London) 432, 197 (2004).

${ }^{36}$ E. Peter, P. Senellart, D. Martrou, A. Lemaître, J. Hours, J. M.
Gérard, and J. Bloch, Phys. Rev. Lett. 95, 067401 (2005).

${ }^{37}$ R. J. Thompson, G. Rempe, and H. J. Kimble, Phys. Rev. Lett. 68, 1132 (1992).

${ }^{38}$ J. McKeever, A. Boca, A. D. Boozer, J. R. Buck, and H. J. Kimble, Nature (London) 425, 268 (2003).

${ }^{39}$ G. Rempe, R. J. Thompson, R. J. Brecha, W. D. Lee, and H. J. Kimble, Phys. Rev. Lett. 67, 1727 (1991).

${ }^{40}$ J. Cardy, Scaling and Renormalization in Statistical Physics, Cambridge Lecture Notes in Physics No. 5 (Cambridge University Press, Cambridge, 1996). 